\title{
Construction of haptic stimulus patterns for use in cross-modal and intramodal matching tasks
}

\author{
DAIYO SAWADA \\ University of Alberta, Edmonton, Alberta T6G 2G5, Canada \\ and \\ RONALD F. JARMAN \\ University of British Columbia, Vancouver, British Columbia V6T IW5, Canada
}

\begin{abstract}
A technique for the construction of haptic stimulus patterns to be used in conjunction with auditory and visual patterns in cross-modal and intramodal matching tasks is described. Special attention is paid to the control of confounding influences arising from the spatiotemporal and the active-passive contrasts.
\end{abstract}

Jarman (1977) described a method for producing auditory stimulus patterns for use in cross-modal and intramodal matching tasks. Further, in recognition of the fact that the temporal nature of the auditory stimulus and the spatial nature of a visual stimulus can confound the comparison of cross-modality and intramodality performance, Jarman, Marshall, and Moore (1979) developed methods for the production of separate visual-spatial (VS) and visual-temporal (VT) stimulus patterns to be paired with the auditory-temporal (AT) patterns. In all, these researchers produced nine matching tasks in which an initial or standard stimulus is followed by a matching or comparison stimulus: AT-AT, AT-VS, AT-VT; VS-AT, VS-VS, VS-VT; VT-AT, VT-VS, VT-VT.

The purpose here is to describe further developments in techniques of constructing cross-modal and intramodal tasks, in the form of haptic procedures that are compatible with the auditory and visual procedures developed previously.

\section{CONSTRAINTS ON TEST CONSTRUCTION}

A convenient way to describe the construction of the haptic stimulus patterns is in terms of the constraints imposed on the patterns in view of (1) compatibility with auditory and visual patterns in terms of (a) linearity and (b) signal form and (2) the confounding effect of the (a) spatial-temporal dimension, (b) active-passive dimension, and (c) simultaneous and/or successive processing dimension.

\section{Compatibility}

Linearity. While most haptic stimulus patterns used

This research was supported by Grant $410-78-0013$ from the Social Sciences and Humanities Research Council of Canada (SSHRCC). in cross-modal or intramodal matching tasks to date have used two-dimensional shapes (e.g., Abravanel, 1972; Cronin, 1973; Millar, 1977; Milner \& Bryant, 1970; Zung, 1971) or three-dimensional forms (e.g., Abravanel, 1972; Smith \& Tunick, 1969; Stanley, Kaplan, \& Poole, 1975; Tyrrell, 1977), compatibility with the Jarman (Jarman et al., 1979) auditory and visual stimulus patterns demands a one-dimensional haptic pattern that is linear in either a temporal sense or a spatial sense. Electronic devices have been developed to deliver tactual patterns that are linear in a temporal sense (Gescheider \& Niblette, 1967; Lechelt, 1975), but these devices provide no options for spatial linearity, nor do they take account of the confounding effects of the active-passive dimension. Bickersteth (1979) developed an electronic device for delivering haptic patterns that, although linear in both a temporal and a spatial sense, did not make provision for the active-passive dimension.

Signal form. Jarman's (1977) VS signal consisted of dots interspaced with either short or long gaps to form a linear array of dots (e.g., $00000 \mathrm{0}$ ). The VT signal consisted of light flashes each of $.15 \mathrm{sec}$ duration separated by short $(.35-\mathrm{sec})$ pauses or long $(1.35-\mathrm{sec})$ pauses to form a linear sequence of flashes. The AT signal was formed from $1,000 \cdot \mathrm{Hz}$ tones, with duration and pausing the same as for the VT signal described above. The three stimulus patterns (VS, VT, AT) were delivered by slides, a flashing light, and a tape recorder, respectively. The common characteristics of the signal forms were (1) discrete individual signals (2) separated by short or long gaps.

\section{Confounding Effects}

Spatial-temporal dimension. Much of the early research using the modality matching paradigm involving the auditory and visual modalities failed to recognize that 
differences between cross-modal (A-V or $\mathrm{V}-\mathrm{A}$ ) and intramodal (A-A or V-V) performances were confounded with spatial-temporal differences in that the auditory stimulus patterns were temporal in nature, whereas the visual stimulus patterns were spatial in nature. Consequently, the cross-modal performances involved a temporal-spatial transformation, whereas the intramodal performances did not (Sterritt, Martin, \& Rudnick, 1971). Jarman et al. (1979) provided control over this confounding effect by developing two versions of the visual stimulus: a VS and a VT configuration. The confounding effect of the spatial-temporal dimension could thus be assessed by comparing, for example, the cross-modal performance AT-VS with the cross-modal performance AT-VT.

Like the visual stimulus patterns, haptic stimulus patterns can be presented temporally (components of the stimulus are presented in serial order) or spatially (all the components of the stimulus are presented at the same time). Thus, comparisons involving the haptic modality can suffer the same spatial-temporal confounding as occurred with the visual modality. The effect of such confounding is apparently significant: Cronin (1973) presented evidence showing systematic differences between haptic-spatial and haptic-temporal performances. Accordingly, the spatial-temporal dimension plays a critical role in the design of the procedures described here.

Active-passive dimension. Procedures for presenting haptic stimulus patterns are characteristically of two types: (1) those that permit subjects to actively search the pattern and (2) those that force the subject to be a passive receiver of the stimulus. As might be expected, the differences in the effect of these two procedures can be quite dramatic (Lackner, 1977). More critically, there is a tendency for the active-passive dimension to be confounded with the spatial-temporal dimension. For example, the temporal tasks (AT or VT) of Jarman et al. (1979) are both passively received. On the other hand, their spatial task (VS) is actively searched. Thus, the spatial-temporal dimension and the active-passive dimension are confounded in these tasks. The procedures described in the present paper are explicitly designed to control for this confounding.

Simultaneous and/or successive processing dimension. The notions of simultaneous and successive processing have proved potent in information processing theory (Das, Kirby, \& Jarman, 1975). These same notions play a key role in the design of the proposed procedures to control the effects of the active-passive and the spatial-temporal confounding. In particular, temporal sequencing demands successive acquisition of information. Central in this sequence of perception is the fact that no two signals are perceived together except as made possible by immediate memory. On the other hand, spatial sequencing permits simultaneous as well as sequential acquisition of information. The characterization of the spatial sequence as allowing either a successive or a simultaneous processing strategy (or indeed, the use of both) and of the temporal sequence as allowing only a successive strategy contains the basis for the operational solution to the control of spatial-temporal/active-passive confounding.

The implications of the constraints discussed in this section are that (1) the haptic patterns must be linear, (2) each haptic signal must be discrete, with patterns determined by short and long gaps, (3) the effects of the active-passive dimension must not be confounded with the effects of the spatial-temporal dimension, (4) the effects of the spatial-temporal dimension must not be confounded with the cross-/intramodal dimension, and (5) the auditory pattems are essentially temporally and passively received. Control of these constraints must therefore be dealt with by carefully designing the haptic instrument.

\section{TEST CONSTRUCTION}

\section{Haptic Stimulus Patterns}

The basic haptic signal was provided by the head of map tack pin of 4-mm diameter with a shank of $12 \mathrm{~mm}$. The pins were pressed into the narrow edge of a rectangular rod of sanded Philippine mahogony $1 \times 2 \times 50 \mathrm{~cm}$. Repeated acquisition of discrimination data from children aged 6,7 , and 8 years resulted in the eventual specification of short gaps at $7.5 \mathrm{~mm}$ on center and long gaps at $20 \mathrm{~mm}$ on center. These specifications resulted in haptic stimulus patterns ranging in length from $2.25 \mathrm{~cm}$ to $10.75 \mathrm{~cm}$, a range well within the hand span of young children. Both the initial or standard haptic stimulus pattern and the matching or comparison haptic stimulus pattern are placed on the same rod, the comparison pattern always starting $15 \mathrm{~cm}$ beyond the start point of the initial pattern. Thirty-five such rods were constructed, corresponding to the 35 items in the Jarman (1977) battery of auditory-visual tests. Figure 1 shows a schematic of Rod 20.

A wooden tray with the dual purpose of storing and presenting the rods was constructed. A slot along the leading edge of the tray allows the exposure of the rods one at a time. After exposure, the leading rod can be lifted from the slot and reinserted at the back of the tray and the set of rods can be pushed forward toward the slot so that the next rod is available for exposure. The rods can be slid left or right $15 \mathrm{~cm}$ within the tray to expose either the initial or the comparison pattern as desired. A carrel with a port fitted with double cuffs

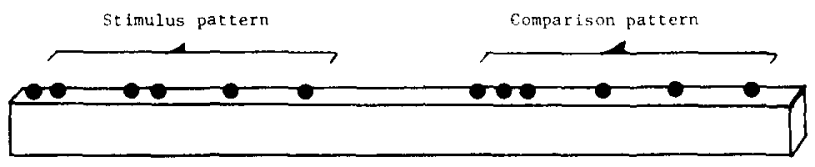

Figure 1. Schematic of Rod 20. 
determines both the seating and hand position of the subject, who places his/her left hand through the cuffs with fingers eventually coming to rest on a small sanded cube of wood fastened just over the exposure slot. To ensure proper alignment, the carrel and tray are connected with a common underlay. For purposes of efficiency, the carrel and tray combination consists of four carrels connected with a common underlay to a single long tray containing long rods $(185 \mathrm{~cm})$, each with four sets of haptic patterns, one set for each carrel. Such an arrangement makes it possible to test subjects four at a time, an important consideration given the repeated-measures design of most modality matching studies. The tray measures $45 \mathrm{~cm}$ wide, $6 \mathrm{~cm}$ high, and $210 \mathrm{~cm}$ long. Two research assistants are required to operate the four-carrel system. One assistant mechanically manipulates the rod-tray system, and the other monitors and controls the subjects' exploration of the beads according to instructions.

\section{Administering the Haptic Patterns}

The Jarman et al. (1979) solution to control spatialtemporal confounding was to develop a VT test and a VS test, each utilizing a different presentation system (different hardware). In the interests of economy and operational efficiency, it is advantageous if the control of confounding variables can be accomplished by software changes as opposed to hardware changes. Accordingly, the solution to the control of the confounding factors of the (1) spatial-temporal dimension and (2) active-passive dimension takes the form of the specification of four software programs, described below. Each is deliverable with the same hardware configuration.

(1) Haptic (temporal-passive) (HTP)-On cue, the subject shifts one finger, preferably the index finger, from the guide cube to the stimulus rod. At a set pace, the experimenter slides the rod along the slot, causing the subject's stationary finger to receive the beads and the gaps one after the other. (2) Haptic (temporalactive) (HTA)-On cue, the subject lowers one finger from the guide cube to the stimulus rod. $\mathrm{He} / \mathrm{she}$ then traverses the beads and gaps in any way and at any rate using only one finger. (3) Haptic (spatial-passive) (HSP)-On cue, the subject lowers his/her fingers from the guide cube to the stimulus pattern. Once the fingers are located to cover the entire haptic array, they remain stationary. (4) Haptic (spatial-active) (HSA)-On cue, the subject lowers his fingers from the guide cube to the haptic pattern. $\mathrm{He} / \mathrm{she}$ then searches the beads and gaps in any way and at any rate using any or all fingers.

Any one of the above four haptic programs can be combined with any of the auditory or visual programs of Jarman et al. (1979) or combined with another haptic program to form a matching task. A cassette tape is required for each pairing to provide for the controlled interaction of the subject with the stimulus patterns. The basic format of the tapes is as described by
Table 1

Recorded Script for Item 6 From the (AT-HSA) Task

"Number 6."

"Ready?"

(Auditory stimulus pattern) $\quad 1.5 \mathrm{~s}$

"Feel."

(Haptic stimulus pattern)

"Lift."

(S lifts hand from beads)

"Answer."

12.0

"Number 7."

(S circles "same" or "different" on an answer sheet)

etc.

Jarman (1977): The first five items are presented with a full set of cues to guide and orient the subject to the task. By Item 6 , the cues have been reduced to bare essentials and are used uniformly thereafter. The recorded script for Item 6 from the (AT-HSA) task, for example, is shown in Table 1.

To date, the nine modality matching tasks possible from using each of the AT, VA, or HSA programs in the initial or the comparison position have been produced and field tested with over 300 subjects ranging in age from 6 to 9 years. Some results in regards to mathematics learning have been obtained showing that matching tasks involving the haptic modality are significantly better predictors of mathematics learning than are matching tasks not involving the haptic modality (Sawada, in press).

\section{REFERENCES}

Abravanel, E. Short-term memory for shape information processed intra- and intermodally at three ages. Perceptual and Motor Skills, 1972, 35, 419-425.

Cronin, V. Cross-modal and intramodal visual and tactual matching in young children. Developmental Psychology, 1973, 8, 336-340.

Bickensteth, P. A cross-cultural study of memory and reasoning. Unpublished doctoral dissertation, University of Alberta, 1979.

Das, J. P., Kirby, J., \& Jarman, R. F. Simultaneous and successive synthesis: An alternative model for cognitive abilities. Psychological Bulletin, 1975, 82, 87-103.

Gescheiden, G. A., \& Niblette, R. K. Cross-modality masking for touch and hearing. Journal of Experimental Psychology, $1967,74,313-320$.

JARMAN, R. F. A method of construction of auditory stimulus patterns for use in cross-modal and intramodal matching tests. Behavior Research Methods \& Instrumentation, 1977, 9, 22-25.

JarMan, R. F., Marshall, M. F., \& MoORs, T. S. Construction of a tape-controlled system of presenting visual-spatial and visual-temporal stimulus patterns for use in cross-modal and intramodel matching tests. Behavior Research Methods \& Instrumentation, 1979, 11, 363-365.

LACKNER, J. R. Adaptation to visual and proprioceptive rearrangement: Origin of the differential effectiveness of active and passive movements. Perception \& Psychophysics, 1977, 21, 55-59.

LECHELT, E. C. Temporal numerosity discrimination: Intermodal comparisons revisited. British Journal of Psycholozy. 1975, C5, 101-108.

Mrlnar, S. Early stages of tactual matching. Penception, 1977, 6, 333-353. 
Milner, A. D., \& Bryant, P. E. Cross-modal matching by young children. Journal of Comparative and Physiological Psychology, 1970, 71, 453-458.

SawadA, D. Multisensory information matching ability and mathematics learning. Journal for Research in Mathematics Education, in press.

SMith, J., \& TuNick, J. Cross-modal transfer of a discrimination by retarded children. Journal of Experimental Child Psychology, $1969,7,274-281$.

Stanley, G., Kaplan, I., \& Poole, C. Cognitive and nonverbal perceptual processing in dyslexics. Journal of General Psychology, 1975, 93, 67-72.
Sterritt, G. M., Martin, V., \& Rudnick, M. Auditory-visual and temporal-spatial integration as determinants of test difficulty. Psychonomic Science, 1971, 23, 289-291.

Tyrrell, D. J. Dimensional effects in cross-modal transfer of discrimination learning in children. Child Development, 1977, 48, 625-629.

ZuNG, B. J. Cross-modal matching among normal and retarded children. Child Development, 1971, 42, 1614-1618.

(Received for publication February 19, 1982; revision accepted May 7, 1982.) 